T. Sunada

Nagoya Math. J.

Vol. 64 (1976), 159-175

\title{
HOLOMORPHIC MAPPINGS INTO A COMPACT QUOTIENT OF SYMMETRIC BOUNDED DOMAIN
}

\author{
TOSHIKAZU SUNADA
}

\section{Introduction}

In this paper, we shall be concerned with the finiteness property of certain holomorphic mappings into a compact quotient of symmetric bounded domain.

Let $\mathfrak{D}$ be a symmetric bounded domain in $n$-dimensional complex Euclidean space $C^{n}$ and $\Gamma \backslash \mathfrak{D}$ be a compact quotient of $\mathfrak{D}$ by a torsion free discrete subgroup $\Gamma$ of automorphism group of $\mathfrak{D}$. Further, we denote by $\ell(D)$ the maximum value of dimension of proper boundary component of $\mathfrak{D}$, which is less than $n$ (=dim $\mathfrak{D})$. Then, the exact statement of our assertion is the following:

THEOREM A. Let $M$ be a compact Kähler manifold. Then there are only finite number of holomorphic mappings of $M$ into $\Gamma \backslash D$ whose rank ${ }^{11}$ are greater than $\ell(D)$. In particular, the set of surjective holomorphic mappings is finite.

Remark. Notice that the compact quotient $\Gamma \backslash D$ is of general type. Hence, in the case of compact quotient Theorem A gives a generalization of a result of S. Kobayashi and T. Ochiai [6] which asserts the finiteness of surjective holomorphic mappings of $M$ onto a compact complex space of general type.

For convenience' sake, we give a complete list of $\ell(D)$ in each irreducible case. For our notation, we refer to S. Helgason [3].

Received March 29, 1976.

1) For a holomorphic mapping $\varphi: M \rightarrow \Gamma \backslash \mathfrak{D}$, rank of $\varphi$ is defined by $\sup _{z \in M}\left(\operatorname{dim}_{z} M-\operatorname{dim}_{z} \varphi^{-1}(\varphi(z))\right)$, 


\begin{tabular}{|c|c|c|}
\hline Domain & Dimension & $\ell(D)$ \\
\hline I $\quad S U(m, n) / S\left(U_{m} \times U_{n}\right)$ & $m \times n$ & $(m-1)(n-1)$ \\
\hline II $S p(n, R) / U(n)$ & $n(n+1) / 2$ & $n(n-1) / 2$ \\
\hline III $S O *(2 n) / U(n)$ & $n(n-1) / 2$ & $(n-2)(n-3) / 2$ \\
\hline IV $S O_{0}(n, 2) / S O(n) \times S O(2)$ & $n$ & 1 \\
\hline $\mathrm{V} \quad E_{6} / S O(10) \cdot S O(2)$ & 16 & 1 \\
\hline VI $E_{7} / E_{6} \cdot S O(2)$ & 27 & 8 \\
\hline
\end{tabular}

From this list, we have

COROLlaRY. Let $\mathfrak{D}$ be the n-dimensional ball (namely $\mathfrak{D}=S U(1, n)$ $\left./ S\left(U_{1} \times U_{n}\right)\right)$. Then, there are only finite number of holomorphic mappings of $M$ into $\Gamma \backslash \mathfrak{D}$ except for constant ones.

We recall that the set of all holomorphic mappings of $M$ into another complex space $N$, which is denoted by $\operatorname{Hol}(M, N)$ in this paper, has a complex analytic space structure with respect to the compact open topology (see A. Douady [1]). Generally, it has many components and is not globally of finite dimension. However, in the course of our proof of Theorem A, we are able to show the following:

THEOREM B. $\operatorname{dim} \operatorname{Hol}(M, \Gamma \backslash D)=\operatorname{dim} \mathfrak{D}$. More precisely, for any non constant mapping $\varphi \in \mathrm{Hol}(M, \Gamma \backslash \mathfrak{D}), \operatorname{dim}_{\varphi} \operatorname{Hol}(M, \Gamma \backslash \mathfrak{D}) \leqq \ell(\mathfrak{D})$.

Remark. Theorem B holds for any torsion free discrete quotient $\Gamma \backslash \mathfrak{D}$ which is not necessarily compact.

\section{Reduction to the linearized problem}

We always assume that $M$ is a compact Kähler manifold.

For a complex manifold $N, T N$ denotes the holomorphic tangent bundle of $N$. Let $\varphi^{-1} T N$ be the induced holomorphic vector bundle on $M$ by a holomorphic mapping $\varphi$ of $M$ into $N, H^{0}\left(M, \varphi^{-1} T N\right)$ the space of holomorphic cross sections of $\varphi^{-1} T N$ which is a finite dimensional vector space over $\boldsymbol{C}$.

A key lemma of our argument is the following which reduces our problem to linearized one.

LEMMA 1. $\operatorname{dim}_{\varphi} \operatorname{Hol}(M, N) \leqq \operatorname{dim} H^{0}\left(M, \varphi^{-1} T N\right)$.

Proof. See M. Namba [7]. Roughly speaking, it uses the fact that 
$\mathrm{Hol}(M, N)$ is realized as a solution space of certain overdetermined nonlinear elliptic equation defined on the space of $C^{\infty}$-mappings of $M$ into $N$, as well as an implicit function theorem of infinite dimensional case. In fact, a germ of $\mathrm{Hol}(M, N)$ at $\varphi$ is realized as a locally closed analytic subset of $H^{0}\left(M, \varphi^{-1} T N\right)$.

Now, in view of Lemma 1 , it is enough to show:

Proposition 1. 1) $\operatorname{dim} H^{0}\left(M, \varphi^{-1} \boldsymbol{T}(\Gamma \backslash \mathfrak{D})\right)=0$ provided that $\operatorname{rank} \varphi$

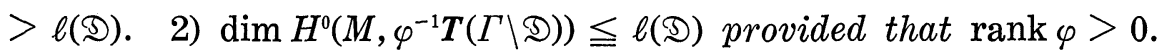

Proof of this proposition will be carried out in later sections. Here we indicate how Proposition 1 implies our theorems: In fact, part 2) of Proposition 1 and Lemma 1 imply that the set $\{\varphi \in \operatorname{Hol}(M, \Gamma \backslash \mathfrak{D})$; $\operatorname{rank} \varphi$ $>\ell(\mathfrak{D})\}$ is discrete in $\mathrm{Hol}(M, \Gamma \backslash \mathfrak{D})$. Since $\Gamma \backslash \mathfrak{D}$ is compact hyperbolic Hol $(M, \Gamma \backslash D)$ is compact (see S. Kobayashi [5]). So the set defined as above has only finite elements, which proves Theorem A. Furthermore, notice that $\varphi$ is non constant if and only if $\operatorname{rank} \varphi>0$. Thus, the second statement of Proposition 1 implies Theorem B.

\section{Holomorphic bisectional curvature}

We return to the general situation. In order to estimate $\operatorname{dim} H^{0}\left(M, \varphi^{-1} \boldsymbol{T} N\right)$ we use the fact that $H^{0}\left(M, \varphi^{-1} T N\right)$ consists of horizontal cross sections with respect to a connection of $\varphi^{-1} T N$. For this purpose, we review the now-standard basic material of hermitian geometry. We make, for convenience in this section, the assumption that $N$ has a Kähler metric $g$, which is of course satisfied in our case $N=\Gamma \backslash \mathfrak{D}$.

We fix a holomorphic mapping $\varphi \in \operatorname{Hol}(M, N)$ and for simplicity in notation let us write $\xi=\varphi^{-1} T N$, which is a holomorphic vector bundle on $M$ of rank $n$ such that the fiber $\xi_{z}$ at $z \in M$ is $T_{\varphi(z)} N$. Let $h$ be an induced hermitian metric of $\xi$ defined by $h_{z}(s, t)=g_{\varphi(z)}(s, t)$ for $s, t \in \xi_{z}$ $\left(=T_{\varphi(z)} N\right)$. We notice that

$$
\left(s_{a}(z)\right)=\left(s_{1}(z), \cdots, s_{n}(z)\right)=\left(\left.\frac{\partial}{\partial w^{1}}\right|_{\varphi(z)}, \cdots,\left.\frac{\partial}{\partial w^{n}}\right|_{\varphi(z)}\right)
$$

is a holomorphic local frame of $\xi$, where $\left(w^{a}\right)=\left(w^{1}, \cdots, w^{n}\right)$ is the local coordinate of $N$.

Here we recall the basic local formulas of hermitian differential 
geometry (refer to V. K. Patodi [8]).

With respect to the local frame $\left(s_{a}\right)$, we put

$$
h_{a b}=h\left(s_{a}, s_{b}\right)
$$

and let $\left(h^{a b}\right)$ be the inverse matrix of $\left(h_{a b}\right)$ so that $\sum h^{a c} h_{c b}=\delta_{b}^{a} \quad\left(\delta_{b}^{a}=1\right.$ or 0 according as $a=b$ or not). Then, the hermitian metric of $\xi$ gives rise to a canonical connection, which is given by

$$
\begin{aligned}
& \nabla_{\alpha}\left(s_{a}\right)=\nabla_{\partial / \partial z^{\alpha}}\left(s_{a}\right)=\sum \ell_{\alpha a}^{b} s_{b}, \\
& \nabla_{a}\left(s_{a}\right)=\nabla_{\partial / \partial z^{\alpha}}\left(s_{a}\right)=0,
\end{aligned}
$$

where $\ell_{\alpha a}^{b}=\sum_{c} \frac{\partial h_{a c}}{\partial z^{\alpha}} h^{c b}$ and $\left(z^{\alpha}\right)=\left(z^{1}, \ldots, z^{m}\right)$ is the local coordinate of M.

Let $K$ be the curvature tensor associated with this hermitian connection. The component $K_{a \alpha \beta}^{b}$ of $K$ are defined by

$$
\sum K_{a \alpha \beta}^{b} s_{b}=K\left(\frac{\partial}{\partial z^{\alpha}}, \frac{\partial}{\partial \bar{z}^{\beta}}\right) s_{a}=\left(\left[\nabla_{\alpha}, \nabla_{\beta}\right]-\nabla_{\left[\partial / \partial z^{\alpha}, \partial / \partial z \beta\right.}\right) s_{a} .
$$

Then

$$
K_{a \alpha \beta}^{b}=-\frac{\partial \ell_{\alpha a}^{b}}{\partial \bar{z}^{\beta}}
$$

In the case of hermitian vector bundle $T N$ with metric $g$, as a rule, $\Gamma$ and $R$ will denote the connection and curvature tensor instead of $\ell$ and $K$. Each component is represented by the local frame $\left(\frac{\partial}{\partial w^{1}}, \cdots, \frac{\partial}{\partial w^{n}}\right)$, namely,

$$
\begin{aligned}
\Gamma_{b c}^{a} & =\sum \frac{\partial g_{c d}}{\partial w^{b}} g^{d a}, \\
R_{b c d}^{a} & =-\frac{\partial \Gamma_{c b}^{a}}{\partial \bar{w}^{d}} .
\end{aligned}
$$

The Kähler property of $g$ is equivalent to the following relation of symmetry :

$$
\Gamma_{b c}^{a}=\Gamma_{c b}^{a}
$$

LEMMA 2. Let $X, Y$ be holomorphic tangent vector at $z$ and $s \in \xi_{z}$. 
Then we have the following relation;

$$
K(X, \bar{Y}) s=R\left(\varphi_{*} X, \overline{\varphi_{*} Y}\right) s .
$$

Proof. By the definition of $h$, it follows that

$$
\begin{aligned}
\ell_{\alpha a}^{b}(z) & =\sum \frac{\partial h_{a d}}{\partial z^{\alpha}}(z) h^{d b}(z)=\sum \frac{\partial g_{a d}(\varphi(z))}{\partial z^{\alpha}} g^{d b}(\varphi(z)) \\
& =\left.\sum \frac{\partial \varphi^{c}}{\partial z^{\alpha}} \frac{\partial g_{a b}}{\partial w^{c}}\right|_{\varphi(z)} g^{d b}(\varphi(z)) \\
& =\sum \frac{\partial \varphi^{c}}{\partial z^{\alpha}} \Gamma_{c a}^{b}(\varphi(z)) .
\end{aligned}
$$

Thus

$$
\begin{aligned}
K_{a \alpha \beta}^{b} & =-\frac{\partial \ell_{\alpha a}^{b}}{\partial \bar{z}^{\beta}} \\
& =-\sum \frac{\partial^{2} \varphi^{c}}{\partial z^{\alpha} \partial \bar{z}^{\beta}} \Gamma_{c a}^{b}(\varphi(z))-\left.\sum \frac{\partial \varphi^{c}}{\partial z^{\alpha}} \frac{\partial \bar{\varphi}^{d}}{\partial \bar{z}^{\beta}} \frac{\partial \Gamma_{c a}^{b}}{\partial \bar{w}^{d}}\right|_{\varphi(z)} \\
& =\sum \frac{\partial \varphi^{c}}{\partial z^{\alpha}} \frac{\partial \bar{\varphi}^{d}}{\partial \bar{z}^{\beta}} R_{a c \bar{d}}^{b},
\end{aligned}
$$

where we use the fact $\varphi$ is holomorphic. This equality proves Lemma 2 .

Finally, we put

$$
R(s, t)=g(R(s, \bar{s}) t, \bar{t}) \quad \text { for } s, t \in \boldsymbol{T N},
$$

which is what is called the holomorphic bisectional curvature determined by $s$ and $t$ (cf. [2]). We notice that

$$
g(R(s, \bar{t}) u, \bar{v})=\overline{g(R(t, \bar{s}) v, \bar{u})},
$$

and the Kähler property means $R(s, t)=R(t, s)$. If we set

$$
R_{a \bar{b} c \bar{d}}=-g_{e b} R_{a c \bar{d}}^{e}=g\left(R\left(\frac{\partial}{\partial w^{c}}, \frac{\partial}{\partial \bar{w}^{d}}\right) \frac{\partial}{\partial \bar{w}^{b}}, \frac{\partial}{\partial w^{a}}\right)
$$

then

$$
R(s, t)=-\sum R_{a b c \bar{d}} t^{a} \bar{t}^{b} s^{c} \overline{\boldsymbol{s}}^{d},
$$

where $s=\sum s^{a} \frac{\partial}{\partial w^{a}}, t=\sum t^{a} \frac{\partial}{\partial w^{a}}$. 


\section{Laplacian}

We introduce a Kähler metric $g^{\prime}$ on $M$, and let $d g^{\prime}$ be the canonical volume element associated with $g^{\prime}$. Then, the Laplacian with coefficient in $\xi$ is as usual defined by $\square=\bar{\partial}^{*} \bar{\partial}: C^{\infty}(\xi) \rightarrow C^{\infty}(\xi)$ where $\bar{\partial}^{*}$ denotes the formal adjoint of first exterior operator $\bar{\partial}: C^{\infty}(\xi) \rightarrow C^{\infty}\left(T^{*} M \otimes \xi\right)$ with respect to the metrices $h, g^{\prime}$. As is well-known, the following are equivalent: For $s \in C^{\infty}(\xi)$

i) $\square s=0$, namely $s$ is harmonic,

ii) $\bar{\partial} s=0$, namely $s$ is holomorphic,

iii) $\nabla_{\bar{X}} s=0$ for any $X \in T M$,

iv) $\int_{M} h(\square s, s) d g^{\prime}=0$.

Moreover, we establish the following lemma as for an expression of the Laplacian in terms of connection and curvature.

Lemma 3. Let $s$ be a $C^{\infty}$-section of $\xi$. Then

$$
-\square s=\sum g^{\prime \beta \alpha} \nabla_{\beta} \nabla_{\alpha} s+\sum g^{\prime \beta \alpha} K\left(\frac{\partial}{\partial z^{\alpha}}, \frac{\partial}{\partial \bar{z}^{\beta}}\right) s \text {. }
$$

Proof. We recall the Laplacian $\square$ is given by

$$
-\square s=\sum g^{\prime \beta \alpha} \nabla_{\alpha} \nabla_{\beta} s,
$$

(see V. K. Patodi [8]). Thus, via the commutation formula $\left(\nabla_{\beta} \nabla_{\alpha}-\nabla_{\alpha} \nabla_{\beta}\right) s$ $=-K\left(\frac{\partial}{\partial z^{\alpha}}, \frac{\partial}{\partial \bar{z}^{\beta}}\right) s$, we have Lemma 3 .

For $s \in C^{\infty}(\xi)$, we define a hermitian form $R_{s}$ on $N$ by

$$
R_{s}(u, \bar{v})=g(R(s, \bar{s}) u, \bar{v}) .
$$

Then we have the following integral formula which plays the essential role in our argument.

LEMMA 4. $\int_{M} h(\square s, s) d g^{\prime}=\int_{M} \sum g^{\prime \beta \alpha} h\left(\nabla_{\alpha} s, \nabla_{\beta} s\right) d g^{\prime}-\int_{M} \operatorname{Tr} \varphi^{*} R_{s} d g^{\prime}$, where $\operatorname{Tr} \varphi^{*} R_{s}$ denotes the trace of a hermitian form $\varphi^{*} R_{s}$ on $M$ with respect to a hermitian metric $g^{\prime}$.

Proof. By the definition of the hermitian connection, we have for $s, t \in C^{\infty}(\xi)$ 


$$
\begin{aligned}
& \frac{\partial}{\partial z^{\alpha}} h(s, t)=h\left(\nabla_{\alpha} s, t\right)+h\left(s, \nabla_{\alpha} t\right), \\
& \frac{\partial}{\partial \bar{z}^{\alpha}} h(s, t)=h\left(\nabla_{\alpha} s, t\right)+h\left(s, \nabla_{\alpha} t\right) .
\end{aligned}
$$

Thus

$$
h\left(\nabla_{\beta} \nabla_{\alpha} s, s\right)=-h\left(\nabla_{\alpha} s, \nabla_{\beta} s\right)+\frac{\partial}{\partial \bar{z}^{\beta}} h\left(\nabla_{\alpha} s, s\right) .
$$

Then we shall show that

$$
\int_{M} \sum g^{\prime \beta \alpha} \frac{\partial}{\partial \bar{z}^{\beta}} h\left(\nabla_{\alpha} s, s\right) d g^{\prime}=0 .
$$

For, putting $\omega=\sum h\left(\nabla_{\alpha} s, s\right) d z^{\alpha} \in C^{\infty}\left(T^{*} M\right)$, we find

$$
\partial^{*} \omega=\sum g^{\prime \beta \alpha} \frac{\partial}{\partial \bar{z}^{\beta}} h\left(\nabla_{\alpha} s, s\right),
$$

where $\partial: C^{\infty}(M) \rightarrow C^{\infty}\left(T^{*} M\right)$ is the exterior differential with respect to $z$, and $\partial^{*}$ is the adjoint of $\partial$. So

$$
\int_{M} \sum g^{\prime \beta \alpha} \frac{\partial}{\partial \bar{z}^{\beta}} h\left(\nabla_{\alpha} s, s\right) d g^{\prime}=\int_{M} \partial^{*} \omega d g^{\prime}=\int_{M} g^{\prime}(\omega, \partial(1)) d g^{\prime}=0 .
$$

Let us now pass to the proof of Lemma.

$$
\begin{aligned}
\int_{M} h(\square s, s) d g^{\prime}= & -\int_{M} h\left(\sum g^{\prime \beta \alpha} \nabla_{\beta} \nabla_{\alpha} s+\sum g^{\prime \beta \alpha} K\left(\frac{\partial}{\partial z^{\alpha}}, \frac{\partial}{\partial \bar{z}^{\beta}}\right) s, s\right) d g^{\prime} \\
= & -\int_{M} \sum g^{\prime \beta \alpha} h\left(\nabla_{\beta} \nabla_{\alpha} s, s\right) d g^{\prime}-\int_{M} \sum g^{\prime \beta \alpha} h\left(K\left(\frac{\partial}{\partial z^{\alpha}}, \frac{\partial}{\partial \bar{z}^{\beta}}\right) s, s\right) d g^{\prime} \\
= & \int_{M} \sum g^{\prime \beta \alpha} h\left(\nabla_{\alpha} s, \nabla_{\beta} s\right) d g^{\prime}-\int_{M} \sum g^{\prime \beta \alpha} \frac{\partial}{\partial \bar{z}^{\beta}} h\left(\nabla_{\alpha} s, s\right) d g^{\prime} \\
& -\int_{M} \sum g^{\prime \beta \alpha} h\left(K\left(\frac{\partial}{\partial z^{\alpha}}, \frac{\partial}{\partial \bar{z}^{\beta}}\right) s, s\right) d g^{\prime} \\
= & \int_{M} \sum g^{\prime \beta \alpha} h\left(\nabla_{\alpha} s, \nabla_{\beta} s\right) d g^{\prime}-\int_{M} \sum g^{\prime \beta \alpha} R_{b c e \bar{d}} s^{b} \bar{s}^{c} \frac{\partial \varphi^{e}}{\partial z^{\alpha}} \frac{\partial \bar{\varphi}^{d}}{\partial \bar{z}^{\beta}} d g^{\prime} .
\end{aligned}
$$

Here we have used above identity and Lemma 2. Since the integrand of the second term of the last quantity is just $\operatorname{Tr} \varphi^{*} R_{s}$, we have eventually Lemma 4.

Now we put the following assumption, which will be later establish- 
ed in the case of discrete quotient $\Gamma \backslash \mathfrak{D}$ with a natural Kähler metric.

(*) $\quad R_{s}(t)=R(s, t) \leqq 0 \quad$ for any $s, t \in T N$.

In such circumstance, we have following important results.

LEMMA 5. If Kähler manifold $(N, g)$ satisfies (*), then i) $C^{\infty}$-cross section $s$ of $\xi\left(=\varphi^{-1} T N\right)$ belongs to $H^{0}(M, \xi)$ if and only if $s$ is horizontal, that is to say, $\nabla_{X} s=\nabla_{\bar{X}} s=0$ for any $X \in T M$, ii) for any $z \in M$, the mapping $\tau_{z}: H^{0}(M, \xi) \rightarrow T_{\varphi(z)} N$ defined by the restriction $s \mapsto s(z)$ is injective,

iii) $\operatorname{Im} \tau_{z}$ is contained in the vector space $\left\{u \in T_{\varphi(z)} N ; R(s, t) u=0\right.$ for any $\left.s, t \in \operatorname{Im} \varphi_{* z}\right\}$.

Proof. By Lemma 4, for $s \in H^{0}(M, \xi)$ we have

$$
0=\int_{M} \sum g^{\prime \beta \alpha} h\left(\nabla_{\alpha} s, \nabla_{\beta} s\right) d g^{\prime}+\int_{M}-\operatorname{Tr} \varphi^{*} R_{s} d g^{\prime}
$$

From our assumption, we obtain $-\operatorname{Tr} \varphi^{*} R_{s} \geqq 0$, so $\int_{M}-\operatorname{Tr} \varphi^{*} R_{s} d g^{\prime} \geqq 0$. On the other hand, $\int_{M} \sum g^{\prime \beta \alpha} h\left(\nabla_{\alpha} s, \nabla_{\beta} s\right) d g^{\prime} \geqq 0$ since the integrand is a non negative function. Thus, if $s$ is a holomorphic section, then necessarily

$$
\int_{M} \sum g^{\prime \beta \alpha} h\left(\nabla_{\alpha} s, \nabla_{\beta} s\right) d g^{\prime}=0
$$

so

$$
\sum g^{\prime \beta \alpha} h\left(\nabla_{\alpha} s, \nabla_{\beta} s\right) \equiv 0 \quad \text { on } M
$$

Thus we have: $\nabla_{\alpha} s=0$ for any $\alpha$. This proves i) (converse is clear). ii) follows immediately from the horizontalness of $s \in H^{0}(M, \xi)$.

To prove iii), we notice that

$$
\left(V_{\alpha} s\right)^{a}=\frac{\partial s^{a}}{\partial z^{\alpha}}+\sum \ell_{\alpha b}^{a} s^{b}=0
$$

Differentiating this equality by $\bar{z}^{\beta}$ and using the fact that $s$ is holomorphic, we have

$$
\sum K_{b \alpha \beta}^{a} s^{b}=K\left(\frac{\partial}{\partial z^{\alpha}}, \frac{\partial}{\partial \bar{z}^{\beta}}\right) s=0 \quad \text { for all } \alpha, \beta .
$$


This completes the proof of iii).

\section{Holomorphic mappings into a Kähler manifold with the property $\left(^{*}\right)$}

Although the present work is devoted primarily to the holomorphic mappings into a compact quotient of symmetric bounded domain, in this section we will consider more general case.

Throughout this section we assume again that $M$ is a compact Kähler manifold and $N$ is a Kähler manifold. We notice that for a holomorphic mapping $\varphi$ of $M$ into $N$

$$
\operatorname{rank} \varphi=\max _{z \in M} \operatorname{rank} \varphi_{* z},
$$

where $\varphi_{* z}: \boldsymbol{T}_{z} M \rightarrow \boldsymbol{T}_{\varphi(z)} N$ denotes the holomorphic differential of $\varphi$ at $z$.

We set: for a vector subspace $V_{p}$ of $T_{p} N$

$$
V \underset{p}{*}=\left\{s \in T_{p} N ; R(u, \bar{v}) s=0 \text { for any } u, v \in V_{p}\right\} \text {. }
$$

Definition. For $k=0,1, \cdots, n=\operatorname{dim} N$ and $p \in N$, let

$$
\ell_{p}(k):=\max \operatorname{dim} V_{p}^{*}
$$

where the maximum is over all $k$-dimensional subspace $V_{p}$ of $T_{p} N$. Furthermore we set

$$
\ell(k)=\max _{p \in M} \ell_{p}(k)
$$

We also put

$$
\operatorname{Hol}_{k}(M, N)=\{\varphi \in \operatorname{Hol}(M, N) ; \operatorname{rank} \varphi \geqq k\} .
$$

Then $\operatorname{Hol}_{k}(M, N)$ is open in $\mathrm{Hol}(M, N)$ and

$$
\operatorname{Hol}_{n}(M, N) \subset \operatorname{Hol}_{n-1}(M, N) \subset \cdots \subset \operatorname{Hol}_{0}(M, N)=\operatorname{Hol}(M, N) .
$$

We notice that $\mathrm{Hol}(M, N)$ contains the set of constant mappings which is a connected component and identified with $N$. We denote by $\operatorname{Hol}(M, N) \backslash N$ the complement of $N$ in $\operatorname{Hol}(\dot{M}, N)$. Then it is obvious that $\operatorname{Hol}_{1}(M, N)=\mathrm{Hol}(M, N) \backslash N$.

Proposition 2. If $(N, g)$ satisfies $\left(^{*}\right)$, then

$$
\operatorname{dim} \mathrm{Hol}_{k}(M, N) \leqq \ell(k) .
$$

Moreover, if $R(s, s) \neq 0$ for $s \neq 0$, then 


$$
\operatorname{dim} \operatorname{Hol}_{k}(M, N) \leqq \operatorname{dim} N-k .
$$

Proof. For $\varphi \in \operatorname{Hol}_{k}(M, N)$, we choose a point $z$ of $M$ such that

$$
k \leqq \operatorname{rank} \varphi=\operatorname{rank} \varphi_{* z} .
$$

By Lemma 1, $§ 2$,

$$
\operatorname{dim}_{\varphi} \operatorname{Hol}(M, N) \leqq \operatorname{dim} H^{0}(M, \xi) .
$$

On the other hand, by Lemma 5, $\S 4$, and the definition of $\ell(k)$

$$
\operatorname{dim} H^{0}(M, \xi)=\operatorname{dim} \operatorname{Im} \tau_{z} \leqq \operatorname{dim}\left(\operatorname{Im} \varphi_{* z}\right)^{*} \leqq \ell_{\varphi(z)}(k) \leqq \ell(k) .
$$

This proves the first statement. The second statement follows from the fact that for any subspace $V_{p} \subset T_{p} N, V_{p}^{*} \cap V_{p}=0$ (for, if $s \in V_{p}^{*} \cap V_{p}$, then $R(s, s)=g(R(s, \bar{s}), \bar{s})=0$. This contradicts our assumption.)

Remark. i) $R(s, s)$ is what is called the holomorphic sectional curvature determined by $s$.

ii) If $R(s, s)<-c<0$ for any unit vector $s$, then $N$ is hyperbolic (see S. Kobayashi [5].)

iii) Connecting with ii) of Lemma 5, $\S 4$, we are able to prove that the set of holomorphic mappings of $M$ into $N$ with the property (*) sending a given point $z \in M$ to a given point $w \in N$ is discrete. Moreover, if $N$ is complete hyperbolic, then it is finite.

Proposition 3. If $(N, g)$ satisfies $\left(^{*}\right)$ and $R(s, s) \neq 0$ for $s \neq 0$, then the set of holomorphic mappings whose rank is equal to $\operatorname{dim} N$ is finite.

Proof. If there is a holomorphic mapping $\varphi$ such that $\operatorname{rank} \varphi=$ $\operatorname{dim} N$, then $N$ is compact and $\varphi(M)=N$, whence we may assume $N$ is compact. By above remark $N$ is hyperbolic. Thus it suffices to show $\mathrm{Hol}_{n}(M, N)$ is discrete. But this is done in Proposition 2.

Proposition 4. If $N$ is compact and $R(s, s)<0$ for any non zero $s, t \in \boldsymbol{T} N$, then $\mathrm{Hol}(M, N) \backslash N$ is finite, namely there are only finite number of holomorphic mappings of $M$ into $N$ except for constant ones.

Proof. This follows at once from the fact: $\ell(1)=0$.

\section{Review of symmetric bounded domain}

According to Lemma 5, we are thus only concerned with the cur- 
vature tensor, now. In this section, we introduce in the usual way a Kähler metric in $\mathfrak{D}$ and $\Gamma \backslash \mathfrak{D}$ which satisfies our assumption $\left(^{*}\right)$ of $\S 4$, and give a brief review of some well-known facts related to boundary components of symmetric bounded domain. We refer to Helgason's book [3] for details.

Let $\mathfrak{S}$ be a symmetric bounded domain, and fix a point $0 \in \mathfrak{S}$. Then $D$ is a hermitian symmetric space of non compact type which is exactly a homogeneous manifold $G / K$, where $G$ is a connected non compact semisimple Lie group and $K$ is a maximal compact subgroup of $G$ that is given as isotropy group at 0 . We denote by $g$ a Lie algebra of $G$ and by $\mathfrak{f}$ the subalgebra of $\mathfrak{g}$ corresponding to $K$. Let $\mathfrak{g}=\mathfrak{f}+\mathfrak{p}$ be a Cartan decomposition of $\mathfrak{g}$ and $\beta$ the Killing form of $\mathfrak{g}$.

If $\mathfrak{z}$ is a Cartan subalgebra of $\mathfrak{l}$ and hence also of $\mathfrak{g}$, then $\mathfrak{g}^{c}$ is a Cartan subalgebra of $\mathrm{g}^{c}$ (complexification of $\mathrm{g}$ ). $\Delta$ will denote the root system of $\mathrm{g}^{c}$ with respect to $\mathfrak{S}^{C}$. We may choose root vectors $e_{\alpha}, \alpha \in \Delta$, and elements $h_{\alpha}$ of $\mathfrak{g}^{c}$ such that

$$
\left[e_{\alpha}, e_{-\alpha}\right]=h_{\alpha}
$$

and

$$
\alpha\left(h_{\beta}\right)=2(\alpha, \beta)(\beta, \beta)^{-1} \quad(\alpha, \beta \in \Delta)
$$

(, ) being the restriction of the Killing form to $\mathfrak{g}^{C}$.

Identifying $\mathfrak{p}$ with $T_{0} \mathfrak{D}$, we give a $G$-invariant hermitian (Kähler) metric $\tilde{g}$ on $\mathfrak{D}$ in the usual way which coincides with restriction of Killing form $\beta$ at 0 , and $\tilde{g}$ allows one to define a Kähler metric $g$ on discrete quotient $N=\Gamma \backslash \mathfrak{D}$.

We notice that $\mathfrak{p}$ has a natural complex structure $J$ derived from that of $T_{0} \supseteqq$ which we recognize by writing

$$
\mathfrak{p} \otimes C=\mathfrak{p}^{+}+\mathfrak{p}^{-}, \mathfrak{p}^{ \pm}: \pm \sqrt{-1} \text {-eigen space of } J,
$$

where $\mathfrak{p}^{+}$is identified with the holomorphic tangent space $\boldsymbol{T}_{0} \mathfrak{D}$. Then

$$
\mathfrak{p}^{+}=\sum_{\alpha \in \Delta_{P}^{+}} \mathfrak{g}^{\alpha},
$$

where $\Delta_{p}^{+}$denotes the set of non compact positive roots with respect to an ordering of $\Delta$.

We shall now describe the curvature of $\mathfrak{D}$ (or $\Gamma \backslash \mathfrak{D}$ ) in group theoretic terms. 
LEMMA 6. Let $\pi: \mathfrak{D} \rightarrow \Gamma \backslash \mathfrak{D}$ be the natural projection, and $R$ the curvature tensor of $\Gamma \backslash \mathfrak{D}$. Then, identifying $\boldsymbol{T}_{\pi(0)}(\Gamma \backslash \mathfrak{D})$ with $\mathfrak{p}^{+}$, we have the following relations.

i) $R_{\pi(0)}(s, \bar{t}) u=-[[s, \bar{t}], u] \quad$ for $s, t, u \in \mathfrak{p}^{+}$,

ii) $R_{\pi(0)}(s, t)=\beta([s, \bar{t}], \overline{[s, \bar{t}]}) \leqq 0$,

iii) $R_{\pi(0)}(s, s)=0$ if and only if $s=0$.

In particular, $(\Gamma \backslash \mathfrak{D}, g)$ satisfies our assumption $(*)$.

Proof. Part i) follows at once from the standard symmetric space theory. For the second part, we recall the property of the Killing form: namely

$$
\begin{aligned}
R_{\pi(0)}(s, t) & =\beta(-[s, \bar{s}] t, \bar{t}) \\
& =\beta([\bar{s}, t] s, \bar{t}) \\
& =\beta([\bar{s}, t],[s, \bar{t}]) \\
& =\beta([s, \bar{t}], \overline{[s, \bar{t}]}) .
\end{aligned}
$$

Since $\beta$ is negative definite on $\mathfrak{l}^{\mathfrak{a}}$ and $[s, \bar{t}]$ is contained in $\mathfrak{f}^{c}$, we have $R(s, t) \leqq 0$. Next, we assume $R(s, s)=0$ for $s=\sum a^{\alpha} e_{\alpha} \in \mathfrak{p}^{+}\left(a_{\alpha} \in C\right)$. Then, as a result of ii) $[s, \bar{s}]=0$. But

$$
[s, \bar{s}]=\left[\sum a^{\alpha} e_{\alpha}, \sum \bar{a}^{\beta} e_{-\beta}\right] \equiv \sum\left|a^{\alpha}\right|^{2} h \quad \bmod \sum_{\alpha \in \Delta} \mathfrak{g}^{\alpha} .
$$

Thus it is necessary that $a^{\alpha}=0$ for all $\alpha \in \Delta$, and so $s=0$. This proves iii).

Now, we review the elementary properties of boundary components. For this purpose, we first recall the Harish-Chandra realization of symmetric bounded domains.

Note that $\mathfrak{p}^{ \pm}$are commutative subalgebras of $\mathrm{g}^{c}$, which give us complex analytic abelian subgroups of $G^{c}$

$$
P^{+}=\exp \mathfrak{p}^{+}, \quad P^{-}=\exp \mathfrak{p}^{-} .
$$

It is well-known, as has been mentioned in Harish-Chandra [4], that the mapping

$$
\eta: \mathfrak{p}^{+} \rightarrow G^{c} / K^{c} P^{-} \quad \text { by } \eta(v)=\exp (v) K^{c} P^{-}
$$

is a complex analytic diffeomorphism of $\mathfrak{p}^{+}$onto a dense open subset of $G^{c} / K^{c} P^{-}$that contains $\mathscr{D}=G / K \subset G^{c} / K^{c} P^{-}$. Thus $\mathfrak{D}$ is canonically embedded in $\mathfrak{p}^{+}$which is called the Harish-Chandra realization of $\mathfrak{D}$. 
In what follows, we assume always that $\mathscr{D} \subset \mathfrak{p}^{+}$is the HarishChandra realization. Let $\bar{D}$ denote the closure of $\mathfrak{D}$ in $\mathfrak{p}^{+}$. Then, we can endow $\bar{D}$ with an equivalence relation by setting $p_{1} \sim p_{2}$ if there is a set $\left\{f_{1}, \cdots, f_{k}\right\}$ of holomorphic mappings of unit disc to $\mathfrak{p}^{+}$with image in $\bar{D}$ such that the image of $f_{j}$ meets the image of $f_{j+1}$ (for $1 \leqq j \leqq$ $k-1$ ), and $p_{1} \in \operatorname{Im} f_{1}$ and $p_{2} \in \operatorname{Im} f_{k}$. The equivalence classes are called the boundary components of $\mathfrak{D}$. $(\mathcal{D}$ itself is an (improper) boundary component and the topological boundary of $\mathscr{D}$ is a disjoint union of (proper) boundary components. By the definition, $\ell(D)$ is equal to the maximum value of dimension of proper boundary component of $\mathfrak{D}$. We refer to J. A. Wolf [9] for various more geometric descriptions of the boundary component.

By using the classification of boundary components, we are able to compute $\ell(D)$ in each irreducible case as given in $\S 1$. It can be also computed more directly by the following identity:

$$
\ell(\mathfrak{D})=\max _{\alpha \in \Delta_{P}^{+}} \#\left\{\beta \in \Delta_{P}^{+} ; \alpha-\beta \notin \Delta\right\} .
$$

The following result is immediate

LEMMA 7. i) $\operatorname{rank} \mathfrak{S} \leqq \ell(\mathfrak{D})<\operatorname{dim} \mathfrak{D}$.

ii) Let $\mathfrak{S}_{1}$ and $\mathfrak{S}_{2}$ be symmetric bounded domains. Then

$$
\ell\left(\mathfrak{D}_{1} \times \mathfrak{D}_{2}\right)=\max \left\{\ell\left(\mathfrak{I}_{1}\right)+\operatorname{dim} \mathfrak{D}_{2}, \ell\left(\mathfrak{D}_{2}\right)+\operatorname{dim} \mathfrak{I}_{1}\right\} .
$$

Next, we introduce the notion of Hermitian symmetric subspace.

Let $\mathfrak{D}_{1}$ be a connected closed complex submanifold of $\mathfrak{D}$. $\mathfrak{D}_{1}$ is said to be a Hermitian symmetric subspace of $\mathscr{D}$ if $\mathfrak{D}_{1}$ is totally geodesic in D. The following lemma is straightforward.

LEMMA 8. If $\mathfrak{D}_{1}$ is a Hermitian symmetric subspace of $\mathfrak{D}$, then $\mathfrak{D}_{1}$ is a Hermitian symmetric space of non compact type.

A (real) subspace $\mathfrak{p}_{1}$ of $\mathfrak{p}$ is called a Lie triple system if $X, Y, Z \in \mathfrak{p}_{1}$ implies $[[X, Y], Z] \in \mathfrak{p}_{1}$. Then we have

LEMMA 9. i) Let $\mathfrak{p}_{1}$ be a J-invariant (namely, $J \mathfrak{p}_{1}=\mathfrak{p}_{1}$ ) Lie triple system contained in $\mathfrak{p}$. Put $\mathfrak{D}_{1}=\operatorname{Exp} \mathfrak{p}_{1}$, where Exp denotes as usual the exponential mapping: $\mathfrak{p} \rightarrow \mathfrak{D}$. Then $\mathfrak{D}_{1}$ is a Hermitian symmetric subspace of $\mathfrak{D}$ satisfying $T_{0} \mathfrak{D}_{1}=\mathfrak{p}_{1}$. 
ii) On the other hand, if $\mathfrak{D}_{1}$ is a Hermitian symmetric subspace of $\mathfrak{D}$ and $0 \in \mathfrak{D}_{1}$, then the subspace $\mathfrak{p}_{1}=T_{0} \mathfrak{O}$ of $\mathfrak{p}$ is a J-invariant Lie triple system and $\mathfrak{S}_{1}=\operatorname{Exp} \mathfrak{p}_{1}$.

Proof. See Helgason's book [3].

LEMMA 10. Let $\mathfrak{p}_{1}$ be a J-invariant subspace of $\mathfrak{p}$. Then $\mathfrak{p}_{1}$ is a Lie triple system of $\mathfrak{p}$ if and only if $\left[\left[\mathfrak{p}_{1}^{-}, \mathfrak{p}_{1}^{+}\right], \mathfrak{p}_{1}^{+}\right] \subset \mathfrak{p}_{1}^{+}$(or equivalently $\left.\left[\left[\mathfrak{p}_{1}^{-}, \mathfrak{p}_{1}^{+}\right], \mathfrak{p}_{1}^{-}\right] \subset \mathfrak{p}_{1}^{-}.\right)$

Proof. Consider the mappings $\tau: \mathfrak{p} \rightarrow \mathfrak{p}^{+}$given by $\tau(X)=X-\sqrt{-1} J X$ and $\operatorname{Re}: \mathfrak{p}^{+} \rightarrow \mathfrak{p}$ by $\operatorname{Re}(s)=s+\bar{s}$. Then, by the elementary computation, we have

$$
[[\tau X, \overline{\tau Y}], \tau Z]=2 \tau([[X, Y], Z]+[[X, J Y], J Z])
$$

and

$$
[[\operatorname{Re} s, \operatorname{Re} t], \operatorname{Re} u]=\operatorname{Re}[[s, \bar{t}], u]+\operatorname{Re}[[s, \bar{t}], \bar{u}] .
$$

Due to these equalities, we get the result.

LEMMA 11. Suppose $\mathfrak{D}$ is embedded in $\mathfrak{p}^{+}$by Harish-Chandra realization. Let $\mathfrak{D}_{1} \subset \mathfrak{D}$ be a Hermitian symmetric subspace of $\mathfrak{D}$ containing 0 , and put $\mathfrak{p}_{1}^{+}=T_{0} \mathfrak{D}_{1}\left(\subset \mathfrak{p}^{+}\right)$. Then $\mathfrak{D}_{1}=\mathfrak{D} \cap \mathfrak{p}_{1}^{+}$and $\mathfrak{D}_{1} \subset \mathfrak{p}_{1}^{+}$is just Harish-Chandra realization of $\mathfrak{D}_{1}$.

Proof. We have the following commutative diagram:

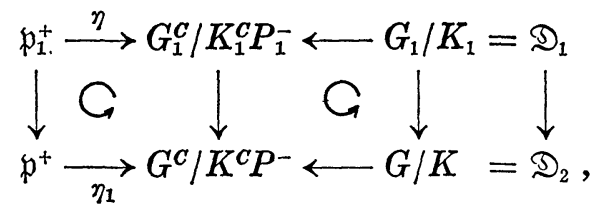

where $G_{1}$ is analytic subgroup of $G$ with Lie algebra $\mathfrak{g}_{1}=\left[\mathfrak{p}_{1}, \mathfrak{p}_{1}\right]+\mathfrak{p}_{1}$. Lemma can be easily derived from this.

In closing this section, we point out the following fact about the boundary component of Hermitian symmetric subspace, which follows from the definition of boundary component, combined with Lemma 11.

LEMMA 12. Let $\mathfrak{D}_{1} \subset \mathfrak{D}$ be a Hermitian symmetric subspace of $\mathfrak{D}$. Then a (proper) boundary component of $\mathfrak{D}_{1}$ is contained in a uniquely determined (proper) boundary component of $\mathfrak{D}$. 


\section{Proof of Proposition 1}

We now come back to the proof of Proposition 1. In order to make sure, we recall the statement of Proposition.

Proposition 1. Let $\varphi$ be a holomorphic mapping of a compact Kähler manifold $M$ into a discrete quotient $\Gamma \backslash \mathfrak{D}$.

i) If rank $\varphi$ is greater than $\ell(\mathfrak{D})$, then $\operatorname{dim} H^{0}\left(M, \varphi^{-1} \boldsymbol{T}(\Gamma \backslash \supseteqq)\right)=0$.

ii) If $\varphi$ is not constant, or equivalently rank $\varphi>0$, then $\operatorname{dim} H^{0}\left(M, \varphi^{-1} T(\Gamma \backslash \supseteqq)\right) \leqq \ell(\supseteqq)$.

First of all, we choose a point $z$ of $M$ so that rank $\varphi_{* z}$ is equal to $\operatorname{rank} \varphi$, and set $V=\operatorname{Im} \varphi_{* z}\left(\subset \boldsymbol{T}_{\varphi(z)}(\Gamma \backslash \mathfrak{D})\right)$. We may assume without loss of generality $\varphi(z)=\pi(0)$, therefore we can regard $V \subset \mathfrak{p}^{+}$. Then in view of Lemma 6, Lemma 5 asserts that $\tau_{z} H^{0}\left(M, \varphi^{-1} T(\Gamma \backslash D)\right)$ is contained in the vector space $\left\{s \in \mathfrak{p}^{+} ;[[u, \bar{v}], s]=0\right.$ for any $\left.u, v \in V\right\}$. For the sake of simplicity, we set for any subspace $V$ of $\mathfrak{p}^{+}$over $C$ :

$$
V^{*}=\left\{s \in \mathfrak{p}^{+} ;[[u, \bar{v}], s]=0 \text { for any } u, v \in V\right\} .
$$

Thus

$$
\operatorname{dim} H^{0}\left(M, \varphi^{-1} \boldsymbol{T}(\Gamma \backslash \mathfrak{D})\right)=\operatorname{dim} \tau_{z} H^{0}\left(M, \varphi^{-1} \boldsymbol{T}(\Gamma \backslash \mathfrak{D})\right) \leqq \operatorname{dim} V^{*} .
$$

Since $\operatorname{dim} V=\operatorname{rank} \varphi$ owing to the choice of $z$, what we must show is: For a subspace $V$ of $\mathfrak{p}^{+}$,

1) $\operatorname{dim} V^{*}=0$ in the case $\operatorname{dim} V>\ell(\mathfrak{D})$,

2) $\operatorname{dim} V^{*} \leqq \ell(\mathfrak{)})$ in the case $\operatorname{dim} V>0$.

We first prove

LEMMA 13. i) $V^{*}=\left\{s \in \mathfrak{p}^{+} ;[\bar{v}, s]=0\right.$ for any $\left.v \in V\right\}$.

ii) $V^{*} \cap V=(0)$ and $V^{* *} \supset V$.

iii) $\operatorname{Re} V^{*}$ and $\operatorname{Re}\left(V^{*}+V^{* *}\right)$ are J-invariant Lie triple system of $\mathfrak{p}$.

Proof. In fact, the equality $[[u, \bar{v}], s]=[u,[\bar{v}, s]]$ implies

$$
V^{*} \supset\left\{s \in \mathfrak{p}^{+} ;[\bar{V}, s]=0\right\} \text {. }
$$

On the other hand, suppose $[[u, \bar{v}], s]=0$ for any $u, v \in V$. Then

$$
0=\beta(-[[v, \bar{v}], s], \bar{s})=\beta([\bar{v}, s],[\overline{\bar{v}, s]}) .
$$

So $[\bar{v}, s]=0$. This establishes i). ii) is obvious. For iii), it is enough to show that 


$$
\begin{aligned}
& {\left[\left[\left[V^{*}, \bar{V}^{*}\right], V^{*}\right], V\right]=0,} \\
& {\left[\left[V^{*}+V^{* *}, \bar{V}^{*}+V^{* *}\right], V^{*}+V^{* *}\right] \subset V^{*}+V^{* *} .}
\end{aligned}
$$

But these follow immediately from Jacobi's identity.

LEMMA 14. We set $\mathfrak{D}_{1}=\operatorname{Exp} \operatorname{Re} V^{*}, \mathfrak{D}_{2}=\operatorname{Exp} \operatorname{Re} V^{* *}$ and $\mathfrak{D}_{0}=$ $\operatorname{Exp} \operatorname{Re}\left(V^{*}+X^{* *}\right)$. Then $\mathfrak{D}_{0}$ is biholomorphic and isometric to $\mathfrak{D}_{1} \times \mathfrak{D}_{2}$.

Proof. We shall show that the mapping $f: \mathscr{D}_{1} \times \mathfrak{D}_{2} \rightarrow \mathfrak{D}_{0}$ given by $\left(\operatorname{Exp} \operatorname{Re} v_{1}, \operatorname{Exp} \operatorname{Re} v_{2}\right) \rightarrow \operatorname{Exp} \operatorname{Re}\left(v_{1}+v_{2}\right)\left(v_{1} \in V^{*}, v_{2} \in V^{* *}\right)$ is a holomorphic isometry. It is easy to find that $f$ is diffeomorphic and isometric. To see the analyticity of $f$, we notice that

$$
\operatorname{Exp} \operatorname{Re}\left(v_{1}+v_{2}\right)=\exp \operatorname{Re} v_{1} \operatorname{Exp} \operatorname{Re} v_{2}=\exp \operatorname{Re} v_{2} \operatorname{Exp} \operatorname{Re} v_{1}
$$

since $\left[\operatorname{Re} v_{1}, \operatorname{Re} v_{2}\right]=0$ and $\operatorname{Exp} X=\exp X \cdot 0$ for $X \in \mathfrak{p}$. Thus, if we denote by $f_{i}: \mathfrak{D}_{i} \rightarrow \mathfrak{D}(i=1,2)$ the natural injections, then

$$
\begin{aligned}
& f\left(w_{1}, \operatorname{Exp} \operatorname{Re} v_{2}\right)=\exp \operatorname{Re} v_{2} \cdot f_{1}\left(w_{1}\right), \\
& f\left(\operatorname{Exp} \operatorname{Re} v_{1}, w_{2}\right)=\exp \operatorname{Re} v_{1} \cdot f_{2}\left(w_{2}\right) .
\end{aligned}
$$

This proves $f$ is holomorphic.

Returning now to Proposition 1, we first note that we can find a boundary component $F_{i}$ of $\mathscr{D}_{0}$ which is biholomorphic to $\mathfrak{D}_{i}(i=1,2)$. In fact, it is sufficient to set $F_{1}=\mathfrak{D}_{1} \times p_{2}$ (resp. $F_{2}=p_{1} \times \mathfrak{D}_{2}$ ) where $p_{i}$ $\in \partial \mathscr{D}_{i}$ is a point in the Silov boundary of $\mathscr{D}_{i}$. From Lemma 12, it now follows that $F_{i}$ is contained in a proper boundary component of $\mathscr{D}$ if and only if $F_{i}$ is a proper one of $\mathfrak{D}_{0}$ or equivalently $\mathfrak{D}_{i}$ is a proper direct product factor of $\mathfrak{D}_{0}$. In terms of $V^{*}, V^{* *}$, it says

a) $\operatorname{dim} V^{* *} \leqq \ell(\mathfrak{D})$ if and only if $\operatorname{dim} V^{*}>0$ (in the case $i=2$ ),

b) $\operatorname{dim} V^{*} \leqq \ell(\mathfrak{D})$ if and only if $\operatorname{dim} V^{* *}>0$ (in the case $i=1$ ). Since $V$ is contained in $V^{* *}$, a) (resp. b)) implies 1) (resp. 2)).

\section{REFERENCES}

[1] A. Douady: Le problème des modules pour les sous-espaces analytiques compacts d'un espace analytique donné, Ann. Inst. Fourier, Grenoble 16 (1966), 1-95.

[2] S. I. Goldberg and S. Kobayashi: Holomorphic bisectional curvature, J. Differential Geometry 1 (1967), 225-233.

[ 3 ] S. Helgason: Differential Geometry and Symmetric Spaces, New York, Academic Press (1962).

[4] Harish-Chandra: Representations of semi-simple Lie group VI, Amer. J. Math. 78 (1956), 564-628. 
[5] S. Kobayashi: Hyperbolic Manifolds and Holomorphic Mappings, Marcel Dekker, 1970.

[6] S. Kobayashi and T. Ochiai: Meromorphic mappings onto compact complex spaces of general type, Inventions Math. 31 (1975), 7-16.

[7] M. Namba: On maximal family of compact complex submanifolds of complex manifolds, Tôhoku Math. J. 24 (1972), 581-609.

[8] V. K. Patodi: An analytic proof of Riemann-Roch-Hirzebruch Theorem for Kaehler manifolds, J. Differential Geometry 5 (1971), 251-283.

[9] J. A. Wolf: Fine structure of hermitian symmetric spaces, Symmetric Spaces (short courses presented at Washington University), Marcel Dekker, 1972.

University of Tokyo 